\title{
Affordable Dual-Sensing Proximity Sensor for Touchless Interactive Systems
}

\author{
Joanna M. Nassar, Marlon C. Diaz and Muhammad M. Hussain* \\ Integrated Nanotechnology Lab (INL) and Integrated Disruptive Electronic Applications (IDEA) Lab, \\ Computer Electrical Mathematical Science and Engineering Division, King Abdullah University of Science and \\ Technology, Thuwal 23955-6900, Saudi Arabia \\ Email: muhammadmustafa.hussain@kaust.edu.sa
}

\begin{abstract}
We report an ultra-low cost flexible proximity sensor using only off-the-shelf recyclable materials such as aluminum foil, napkin and double-sided tape. Unlike previous reports, our device structure exhibits two sensing capabilities in one platform, with outstanding long detection range of $20 \mathrm{~cm}$ and pressure sensitivity of $0.05 \mathrm{kPa}^{-1}$. This is the first ever demonstration of a low-cost, accessible, and batch manufacturing process for pressure and proximity sensing on a singular platform. The mechanical flexibility of the sensor makes it possible to mount on various irregular platforms, which is vital in many areas, such as robotics, machine automation, vehicular technology and inspection tools.
\end{abstract}

\section{Introduction}

Proximity detection is increasingly gaining interest for future interactive technologies. Maturity of this field reveals great opportunities for motion-based interactive systems in human-machine interfacing. However, a key challenge in this technology is cost and size versus detection range. Current developments in proximity sensors are either limited by high cost, such as photoelectric sensing $[1,2]$, or by sensing range dictated by size for capacitive and inductive sensing [3-5]. The new approach we are showing overcomes the size and cost issues while preserving an outstanding detection range of $20 \mathrm{~cm}$ through inductive sensing. But also our device shows multifunctionality with the ability to detect force. The demonstrated fabrication method is affordable, accessible and easily scalable for array integration and reduced manufacturing cost.

\section{Experimental Process}

Sensor structure is schematically illustrated in Fig. 1. It consists of a $1 \times 1 \mathrm{~cm}$ parallel-plate capacitor design, using aluminum foil ( $15 \mu \mathrm{m}$ thick) for electrodes, and an insulation layer consisting of $90 \mu \mathrm{m}$ airgap and $600 \mu \mathrm{m}$ cleanroom napkin, shown in cross-section SEM in Fig. 2. The dielectric material choice enables enhanced compressibility under load, improving pressure detection. The paramagnetic property of the aluminum foil houses induced eddy currents, thus upon application AC signal, aluminum foil generates a local magnetic field. Thus, conductive objects in proximity decrease the charges flux around the capacitor due to shielding effect or induced lossy eddy currents.

\section{Device Characteristics}

Using a $1 \mathrm{MHz}$ modulation frequency, we compare real-time proximity detection of diverse conductive and insulating objects in the XY-plane of the sensor (Fig. 3). Results show no response to a paper in proximity, but high sensitivity to human hand with the fastest detection rate with $\tau=2.88 \mathrm{~s}$. Fig. 4 shows the proximity profile versus discrete distances, where human body is recognized at $20 \mathrm{~cm}$ detection range. In the XZ-plane of the sensor, detection range decreases to $16 \mathrm{~cm}$ (Fig. 5), due to the reduced electromagnetic field profile. Finally, we show pressure detection with a sensitivity of $\sim 0.05 \mathrm{kPa}^{-1}$ in the low-pressure regime (Fig. 6). This is the first time ever proximity and pressure sensing features are reported with such an affordable fabrication technique, while preserving good pressure sensitivity and higher proximity detection range than reported in literature $[3,6]$.

\section{Conclusion}

We have presented a low-cost paper based proximity and pressure sensing features using a singular flexible platform. We show improved proximity detection ranges with respect to size and cost, as well as multifunctionality advantage. Also, we display the benefit of ultra-low cost, accessibility and scalability of our fabrication process.

\section{References}

[1] B. Lukas et al., Org. Electron.,vol. 7, pp. 114-120, (2006); [2] W. Jae-Yeon et al., IEEE Trans. Ind. Electron., vol. 62, p. 536544, (2015); [3] C. Zhenhai et al., IEEE Trans. Ind. Electron., vol. 45, p. 886-894, (1998); [4] L. Hyung-Kew et al., IEEE Sens. J., vol. 9, p. 1748-1755, (2009); [5] K. Pavel et al., Sens. Actuators, A, vol. 110, p. 93-97, (2004); [6] T. Kuniharu et al., Nat. Mater., vol. 9, p. 821-826, (2010). 


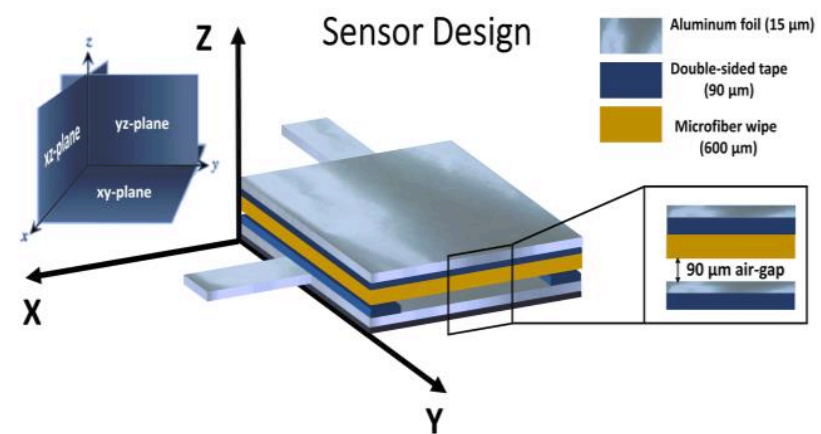

Fig. 1. 3D schematic of the sensor structure and process fabrication. Inset on the right shows the respective layers arrangement in cross-section view.

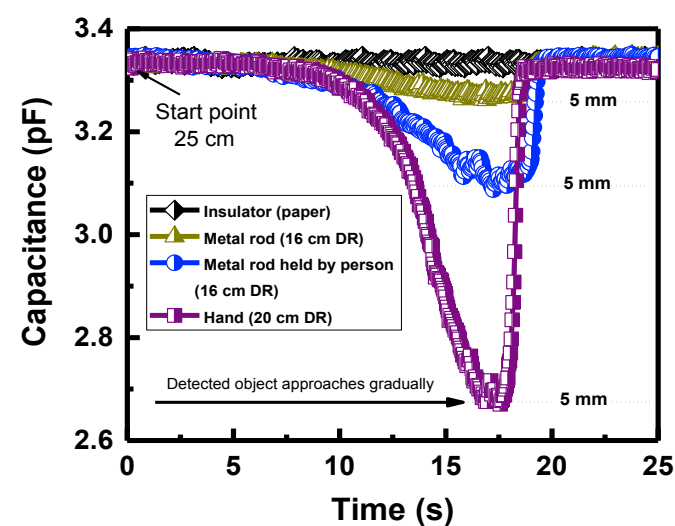

Fig. 3. Real-time XY-plane proximity sensing (zdirection). Plots of various types of detected objects as they approach the sensor at constant rate.

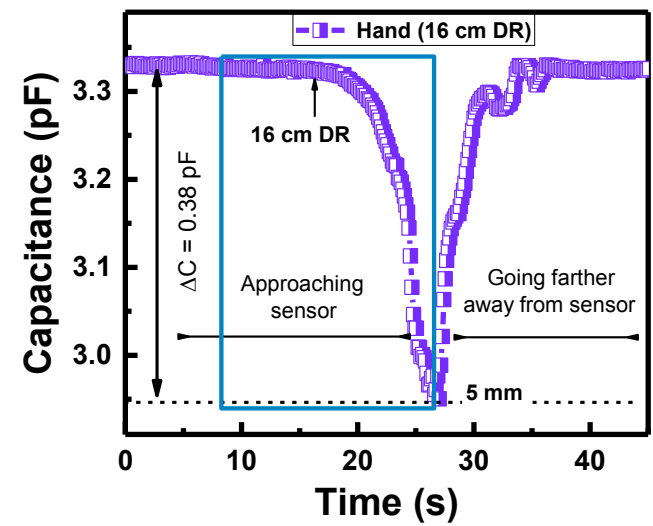

Fig. 5. Real-time XZ/YZ-plane proximity detection of human hand with $16 \mathrm{~cm}$ detection range.

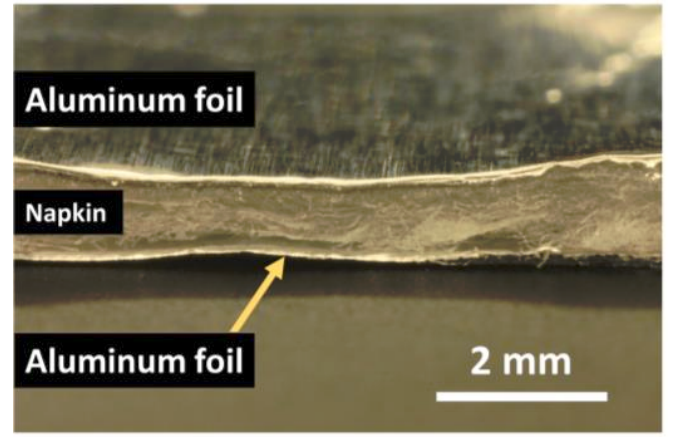

Fig. 2. Digital photo showing the cross-section configuration of the capacitive structure.

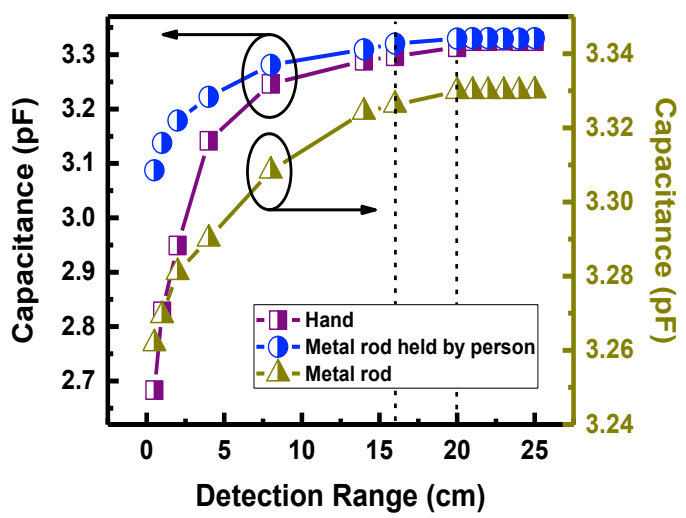

Fig. 4. XY-plane proximity detection with respect to detection range (DR) distance (from $25 \mathrm{~cm}$ to $5 \mathrm{~mm}$ ).

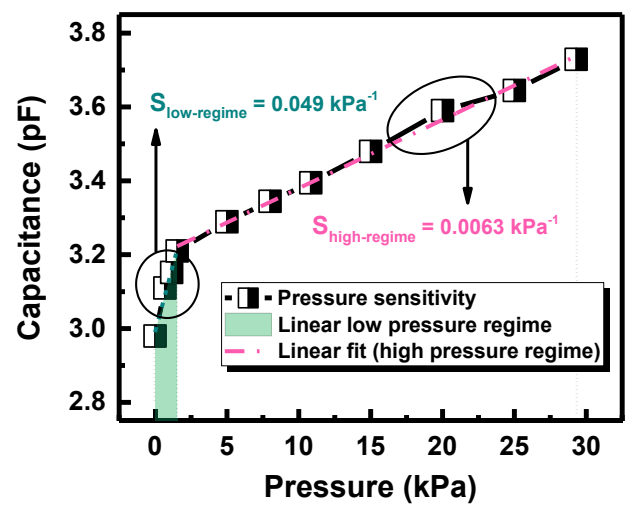

Fig. 6. Pressure sensitivity profile in the low-pressure regime $(<2 \mathrm{kPa})$ and high-pressure regime (up to 30 $\mathrm{kPa})$. 